\title{
Pacific
}

Journal of

Mathematics

\section{ON THE UNIQUENESS OF CAPILLARY SURFACES OVER AN} INFINITE STRIP

JENN-FANG HWANG 


\title{
ON THE UNIQUENESS OF CAPILLARY SURFACES OVER AN INFINITE STRIP
}

\author{
JENN-FANG HWANG
}

\begin{abstract}
In 1987, Tam proved that the solution of the capillary surface equation without gravity over an infinite strip must be a rigid rotation of a cylinder. Here we give a simple proof for Tam's Theorem and generalize his result.
\end{abstract}

1. Introduction. Let $\Omega$ be a domain in $\mathbb{R}^{n}$. Consider the equation of prescribed mean curvature

$$
\operatorname{div} T u=H \text { in } \Omega
$$

where $T u=\frac{D u}{\sqrt{1+|D u|^{2}}}$ and $D u$ is the gradient of $u$.

Finn [4] proved that if $H=n, \Omega$ contains the unit ball $B_{1}$ of $\mathbb{R}^{n}$, and (1) has a solution $u$, then $\Omega$ has to be exactly $B_{1}$ and $u$ must be a lower hemisphere. We emphasize that no boundary condition is imposed.

In the case $\Omega$ is unbounded, Finn [4] conjectured that the only solution of (1) with $H=2$ over an infinite strip of width 1 in $\mathbb{R}^{2}$ is a cylinder. Wang [9] and Collin [2] independently showed that other different solutions can appear, so Finn's conjecture is not true.

In [7], [8] Tam considered the problem related to Finn's conjecture as follows:

$$
\begin{cases}\operatorname{div} T u=H & \text { in } \Omega \\ T u \cdot \nu=\cos \alpha & \text { on } \partial \Omega\end{cases}
$$

where $H$ and $\alpha$ are constants, $\Omega$ is the infinite strip $\left(-\frac{1}{2}, \frac{1}{2}\right) \times \mathbb{R}, \nu$ is the unit outward normal of $\partial \Omega$. The boundary value problem (2) determines the height of a capillary surface without gravity. Note that if (2) has a solution, then $H=2 \cos \alpha([7])$. 
Tam [7] proved that any solution of (2) is of the form $\phi_{\beta}+$ constant for some $|\beta|<1$ if $\frac{\pi}{2}>\alpha>0$, where

$$
\phi_{\beta}=-\frac{1}{\sqrt{1-\beta^{2}}} \sqrt{\left(\frac{1}{2 \cos \alpha}\right)^{2}-x^{2}}+\frac{\beta}{\sqrt{1-\beta^{2}}} y
$$

that is, any solution of (2) must be a cylinder.

The statement above is still true for $\alpha=0$ and was proved by the author first, and then Tam modify his own method in [7] to give a unified proof for all cases $\frac{\pi}{2}>\alpha \geq 0$, (c.f. [8], where the author's name was spelled by Tam as "C. Wong").

In this paper, we do not give our original proof for the case $\alpha=0$, but give a simple proof for the cases $\frac{\pi}{2}>\alpha \geq 0$. We shall prove that if $\int_{\Gamma_{y_{0}}} T u \cdot \nu_{1} d \sigma$ is given where $y_{0}$ is a constant, $\Gamma_{y_{0}}=\left\{\left(x, y_{0}\right) \mid-\frac{1}{2} \leq x \leq \frac{1}{2}\right\}, \nu_{1}=\langle 0,1\rangle$. Then the solution of (2) is unique up to an additive constant. Therefore we not only obtain Tam's Theorem but also generalize it (§2).

The author is grateful to Prof. Finn's kind suggestion on this paper.

2. Simple proof for Tam's Theorem. To simplify the proof for Tam's Theorem, the well-known inequality

$$
(D u-D v) \cdot(T u-T v) \geq \frac{|D u-D v|^{2}}{\max \left(\left(1+|D u|^{2}\right)^{\frac{3}{2}},\left(1+|D v|^{2}\right)^{\frac{3}{2}}\right)}
$$

[6] will be sharpened, and actually we will use the following inequality (3) to deal with integral estimates:

Lemma 1. Let $\Omega \subset \mathbb{R}^{n}$ and let $u, v \in C^{1}(\Omega)$. Then

$$
\begin{aligned}
& (D u-D v) \cdot(T u-T v) \\
& \quad \geq \frac{|D u-D v|^{2}}{\sqrt{1+(|D u|+|D u-D v|)^{2}}}\left(1-\frac{|D u|}{\sqrt{1+|D u|^{2}}}\right) .
\end{aligned}
$$

Proof. Let $|D u|=\alpha$ and $|D u-D v|=b$. After choosing a suitable coordinates, we may assume $D u-D v=(b, 0, \ldots, 0), D u=$ $\left(\alpha \cos \theta, \alpha_{2}, \ldots, \alpha_{n}\right)$ where $0 \leq \theta \leq \pi, \alpha^{2} \cos ^{2} \theta+\alpha_{2}^{2}+\cdots+\alpha_{n}^{2}=\alpha^{2}$. 
Then $D v=\left(\alpha \cos \theta-b, \alpha_{2}, \ldots, \alpha_{n}\right)$. Hence

$$
\begin{aligned}
& \left(\frac{D u}{\sqrt{1+|D u|^{2}}}-\frac{D v}{\sqrt{1+|D v|^{2}}}\right) \cdot(D u-D v) \\
& =\left(\frac{\alpha \cos \theta}{\sqrt{1+\alpha^{2}}}-\frac{\alpha \cos \theta-b}{\sqrt{\left.1+(\alpha \cos \theta-b)^{2}+\alpha_{2}^{2}+\cdots+\alpha_{n}^{2}\right)}}\right) b \\
& =b\left(\alpha \cos \theta\left(\frac{1}{\sqrt{1+\alpha^{2}}}-\frac{1}{\gamma}\right)+\frac{b}{\gamma}\right) \\
& =b\left(\alpha \cos \theta\left(\frac{-2 \alpha b \cos \theta+b^{2}}{\sqrt{1+\alpha^{2}} \gamma\left(\sqrt{1+\alpha^{2}}+\gamma\right)}\right)+\frac{b}{\gamma}\right) \\
& =\frac{b^{2}}{\gamma}\left(\frac{\alpha \cos \theta(-2 \alpha \cos \theta+b)}{\sqrt{1+\alpha^{2}}\left(\sqrt{1+\alpha^{2}}+\gamma\right)}+1\right),
\end{aligned}
$$

where $\gamma=\sqrt{1+\alpha^{2}-2 \alpha b \cos \theta+b^{2}}$.

Since

$$
\begin{aligned}
\sqrt{1+\alpha^{2}}+\sqrt{1+\alpha^{2}-2 \alpha b \cos \theta+b^{2}} & \geq|\alpha|+|\alpha \cos \theta-b| \\
& \geq|-2 \alpha \cos \theta+b|
\end{aligned}
$$

and since $\sqrt{1+\alpha^{2}-2 \alpha b \cos \theta+b^{2}} \leq \sqrt{1+(\alpha+b)^{2}}$, we have

$$
(T u-T v) \cdot(D u-D v) \geq \frac{b^{2}}{\sqrt{1+(\alpha+b)^{2}}}\left(1-\frac{\alpha}{\sqrt{1+\alpha^{2}}}\right)
$$

This completes the proof.

Now we generalize Tam's Theorem.

THEOREM 2. Let $\Omega$ be the infinite strip $\left(-\frac{1}{2}, \frac{1}{2}\right) \times \mathbb{R}$ in $\mathbb{R}^{2}$. Let $u, v$ be $C^{2}(\Omega)$ functions satisfying $T u, T v \in C^{0}(\bar{\Omega}) \cap C^{1}(\Omega)$. Suppose that for every $\varepsilon$ with $0<\varepsilon<\frac{1}{2},|D u|$ is uniformly bounded in $\left[-\frac{1}{2}+\varepsilon, \frac{1}{2}-\varepsilon\right] \times \mathbb{R}$. And if

$$
\begin{cases}\operatorname{div} T v=\operatorname{div} T u & \text { in } \Omega \\ T v \cdot \nu=T u \cdot \nu & \text { on } \partial \Omega \\ \int_{\Gamma_{y_{0}}} T v \cdot \nu_{1} d \sigma=\int_{\Gamma_{y_{0}}} T u \cdot \nu_{1} d \sigma & \text { for some constant } y_{0}\end{cases}
$$

where $\nu$ is the unit outward normal of $\partial \Omega, \nu_{1}=\langle 0,1\rangle, \Gamma_{y_{0}}=\bar{\Omega} \cap$ $\left\{(x, y) \mid y=y_{0}\right\}$, then we have $v \equiv u+$ constant.

REMARK. In (4), we assume neither $\operatorname{div} T u=$ constant nor $T u \cdot \nu=$ constant, hence we generalize Tam's Theorem. 
As for more general domains $\Omega$, the results are stated in Theorem 4.

Proof of Theorem 2. For any two numbers $y_{1}, y_{2}$ with $y_{1}<y_{2}$, we set $\Omega_{y_{1}, y_{2}}=\Omega \cap\left\{(x, y) \mid-y_{1}<y<y_{2}\right\}$. By divergence theorem, we have

$$
\int_{\partial \Omega_{y_{1}, y_{2}}}(T u \cdot \nu-T v \cdot \nu) d \sigma=\iint_{\Omega_{y_{1}, y_{2}}} \operatorname{div} T u-\operatorname{div} T v=0 .
$$

Since $(T u-T v) \cdot \nu=0$ on $\partial \Omega, \nu=\nu_{1}$ on $\Gamma_{y_{2}}$ and $\nu=-\nu_{1}$ on $\Gamma_{y_{1}}$, we obtain

$$
\int_{\Gamma_{y_{2}}}(T u-T v) \cdot \nu_{1} d \sigma-\int_{\Gamma_{y_{1}}}(T u-T v) \cdot \nu_{1} d \sigma=0
$$

Hence

(5)

$$
\int_{\Gamma_{y_{1}}}(T u-T v) \cdot \nu_{1} d \sigma=\text { constant }=\int_{\Gamma_{y_{0}}}(T u-T v) \cdot \nu_{1} d \sigma=0
$$

for every $y_{1} \in \mathbb{R}$.

Similarly, applying divergence theorem again, we have

$$
\begin{aligned}
& \int_{\Gamma_{y_{2}}} \tan ^{-1}(u-v)(T u-T v) \cdot \nu_{1} d \sigma \\
& -\int_{\Gamma_{y_{1}}} \tan ^{-1}(u-v)(T u-T v) \cdot \nu_{1} d \sigma \\
& =\iint_{\Omega_{y_{1}, y_{2}}} \frac{(D u-D v)}{1+(u-v)^{2}} \cdot(T u-T v) \\
& +\iint_{\Omega_{y_{1}, y_{2}}} \tan ^{-1}(u-v)(\operatorname{div} T u-\operatorname{div} T v) \\
& =\iint_{\Omega_{y_{1}, y_{2}}} \frac{(D u-D v)}{1+(u-v)^{2}} \cdot(T u-T v) \geq 0, \quad\left(y_{1}<y_{2}\right) .
\end{aligned}
$$

For each $y \in \mathbb{R}$, let us write

$$
f(y)=\int_{\Gamma_{y}} \tan ^{-1}(u-v)(T u-T v) \cdot \nu_{1} .
$$

By (6), $f(y)$ is increasing in $y$, hence $\lim _{y \rightarrow \pm \infty} f(y)$ exist. We claim that $\lim _{y \rightarrow+\infty} f(y)=0$. Otherwise, there exist two constants $y_{0}^{\prime}$ and $C_{1}$ such that $0<C_{1}<1$ and

$$
|f(y)| \geq C_{1} \text { for every } y \geq y_{0}^{\prime} .
$$


Define $m(y)=\frac{1}{\left|\Gamma_{y}^{\prime}\right|} \int_{\Gamma_{y}^{\prime}} \tan ^{-1}(u-v) d \sigma$ for every $y \geq y_{0}^{\prime}$ where

$$
\Gamma_{y}^{\prime}=\left[-\frac{1}{2}+\frac{C_{1}}{8 \pi}, \frac{1}{2}-\frac{C_{1}}{8 \pi}\right] \times\{y\}
$$

$\left|\Gamma_{y}^{\prime}\right|$ is the length of $\Gamma_{y}^{\prime}$. It follows that $|m(y)| \leq \frac{\pi}{2}$ for every $y \geq y_{0}^{\prime}$. From (5), we see that

$$
\begin{aligned}
& \int_{\Gamma_{y}} m(y)(T u-T v) \cdot \nu_{1} \\
& \quad=m(y) \int_{\Gamma_{y}}(T u-T v) \cdot \nu_{1}=0 \text { for every } y \geq y_{0}^{\prime} .
\end{aligned}
$$

By hypothesis, $|D u|$ is uniformly bounded in

$$
\left[-\frac{1}{2}+\frac{C_{1}}{8 \pi}, \frac{1}{2}-\frac{C_{1}}{8 \pi}\right] \times \mathbb{R}
$$

so there exists a positive constant $C_{2}$ (independent of $y$ ) such that

$$
|D u| \leq C_{2} \text { in }\left[-\frac{1}{2}+\frac{C_{1}}{8 \pi}, \frac{1}{2}-\frac{C_{1}}{8 \pi}\right] \times \mathbb{R} .
$$

Hence for each $y \geq y_{0}^{\prime}$,

$$
\begin{aligned}
& \left|\int_{\Gamma_{y}^{\prime}}\left(\tan ^{-1}(u-v)-m(y)\right)(T u-T v) \cdot \nu_{1}\right| \\
& \quad \geq|| \int_{\Gamma_{y}}\left(\tan ^{-1}(u-v)-m(y)\right)(T u-T v) \cdot \nu_{1} \mid \\
& \quad-\mid \int_{\Gamma_{y} \backslash \Gamma_{y}^{\prime}}\left(\tan ^{-1}(u-v)-m(y)\right)(T u-T v) \cdot \nu_{1} \| .
\end{aligned}
$$

By direct computation, we have

$$
\begin{aligned}
\mid \int_{\Gamma_{y} \backslash \Gamma_{y}^{\prime}}\left(\tan ^{-1}(u-v)-m(y)\right)(T u & -T v) \cdot \nu_{1} \mid \\
& \leq 2 \cdot \frac{C_{1}}{8 \pi}\left(\frac{\pi}{2}+\frac{\pi}{2}\right) \cdot 2=\frac{C_{1}}{2} .
\end{aligned}
$$

Combining this result with (7)-(9) and (11), we have

$$
\begin{aligned}
\left.\mid \int_{\Gamma_{y}^{\prime}} \tan ^{-1}(u-v)-m(y)\right)(T u-T v) & \cdot \nu_{1} \mid \\
& \geq \frac{C_{1}}{2} \text { for every } y \geq y_{0}^{\prime} .
\end{aligned}
$$


Applying Poincaré inequality to the left hand side of (12), we obtain

$$
\begin{aligned}
\frac{C_{1}}{2} & \leq 2 \int_{\Gamma_{y}^{\prime}}\left|\tan ^{-1}(u-v)-m(y)\right| \\
& \leq 2\left|\Gamma_{y}^{\prime}\right| \int_{\Gamma_{y}^{\prime}} \frac{|D u-D v|}{1+(u-v)^{2}} \\
& \leq 2\left|\Gamma_{y}^{\prime}\right|\left(\int_{\Gamma_{y}^{\prime} \cap\left\{|D u-D v|<8^{-1}\left|\Gamma_{y}^{\prime}\right|^{-2} C_{1}\right\}} \frac{|D u-D v|}{1+(u-v)^{2}}\right. \\
& \left.\quad+\int_{\Gamma_{y}^{\prime} \cap\left\{|D u-D v| \geq 8^{-1}\left|\Gamma_{y}^{\prime}\right|^{-2} C_{1}\right\}} \frac{|D u-D v|}{1+(u-v)^{2}}\right) \\
& \leq \frac{C_{1}}{4}+2\left|\Gamma_{y}^{\prime}\right| \int_{\Gamma_{y}^{\prime} \cap\left\{|D u-D v| \geq 8^{-1}\left|\Gamma_{y}^{\prime}\right|^{-2} C_{1}\right\}} \frac{|D u-D v|}{1+(u-v)^{2}} .
\end{aligned}
$$

Hence for every $y \geq y_{0}^{\prime}$, we have

$$
\frac{C_{1}}{8\left|\Gamma_{y}^{\prime}\right|} \leq \int_{\Gamma_{y}^{\prime} \cap\left\{|D u-D v| \geq 8^{-1}\left|\Gamma_{y}^{\prime}\right|^{-2} C_{1}\right\}} \frac{|D u-D v|}{1+(u-v)^{2}} .
$$

From (10), we have

$$
\begin{aligned}
& \frac{|D u-D v|^{2}}{\sqrt{1+(|D u|+|D u-D v|)^{2}}}\left(1-\frac{|D u|}{\sqrt{1+|D u|^{2}}}\right) \\
& \geq \frac{|D u-D v|^{2}}{\sqrt{1+\left(C_{2}+|D u-D v|\right)^{2}}}\left(1-\frac{C_{2}}{\sqrt{1+C_{2}^{2}}}\right) \\
& \geq|D u-D v| \frac{8^{-1}\left|\Gamma_{y}^{\prime}\right|^{-2} C_{1}}{\sqrt{1+\left(C_{2}+8^{-1}\left|\Gamma_{y}^{\prime}\right|^{-2} C_{1}\right)^{2}}}\left(1-\frac{C_{2}}{\sqrt{1+C_{2}^{2}}}\right)
\end{aligned}
$$

in $\Gamma_{y}^{\prime} \cap\left\{|D u-D v| \geq 8^{-1}\left|\Gamma_{y}^{\prime}\right|^{-2} C_{1}\right\}$. Combining (13) with the inequalities (3) and (14), we obtain the estimate

$$
\begin{gathered}
\frac{C_{1}}{8\left|\Gamma_{y}^{\prime}\right|} \leq 8\left|\Gamma_{y}^{\prime}\right|^{2} C_{1}^{-1} \sqrt{1+\left(C_{2}+8^{-1}\left|\Gamma_{y}^{\prime}\right|^{-2} C_{1}\right)^{2}}\left(1-\frac{C_{2}}{\sqrt{1+C_{2}^{2}}}\right)^{-1}- \\
\cdot \int_{\Gamma_{y}^{\prime} \cap\left\{|D u-D v| \geq 8^{-1}\left|\Gamma_{y}^{\prime}\right|^{-2} C_{1}\right\}} \frac{(D u-D v) \cdot(T u-T v)}{1+(u-v)^{2}}
\end{gathered}
$$


Since $\Gamma_{y}^{\prime} \subset \Gamma_{y}$, it is easy to see that

$$
C_{3} \leq \int_{\Gamma_{y}} \frac{(D u-D v) \cdot(T u-T v)}{1+(u-v)^{2}}
$$

for every $y \geq y_{0}^{\prime}$ where

$$
C_{3}=\frac{C_{1}^{2}}{64\left|\Gamma_{y}\right|^{3} \sqrt{1+\left(C_{2}+8^{-1}\left|\Gamma_{y}\right|^{-2} C_{1}\right)^{2}}}\left(1-\frac{C_{2}}{\sqrt{1+C_{2}^{2}}}\right) .
$$

By (6), (7), (15) and Fubini's Theorem, for every $y \geq y_{0}^{\prime}$, we have

$$
\begin{aligned}
& f(y)-f\left(y_{0}\right) \\
& =\int_{\Gamma_{y}} \tan ^{-1}(u-v)(T u-T v) \cdot \nu_{1} \\
& \quad-\int_{\Gamma_{y_{0}^{\prime}}} \tan ^{-1}(u-v)(T u-T v) \cdot \nu_{1} \\
& =\iint_{\Omega_{y_{0}^{\prime}, y}} \frac{(D u-D v)}{1+(u-v)^{2}} \cdot(T u-T v) \\
& =\int_{y_{0}^{\prime}}^{y}\left(\int_{\Gamma_{t}} \frac{(D u-D v)}{1+(u-v)^{2}} \cdot(T u-T v) d \sigma\right) d t \\
& \geq \int_{y_{0}^{\prime}}^{y} C_{3}\left|\Gamma_{t}\right| d t \\
& =C_{3}\left(y-y_{0}^{\prime}\right) .
\end{aligned}
$$

Note that $\left|\int_{\Gamma_{y}} \tan ^{-1}(u-v)(T u-T v) \cdot \nu_{1}\right| \leq \pi \cdot\left|\Gamma_{y}\right|=\pi$. Let $y \rightarrow+\infty$ in (16), we get a contradiction. Thus we obtain $\lim _{y \rightarrow+\infty} f(y)=0$. Similarly, we have $\lim _{y \rightarrow-\infty} f(y)=0$. Using (6) again and letting $y_{2} \rightarrow+\infty, y_{1} \rightarrow-\infty$, we have $\iint_{\Omega} \frac{(D u-D v)}{1+(u-v)^{2}} \cdot(T u-T v)=0$, hence $(D u-D v) \cdot(T u-T v) \equiv 0$ in $\Omega$, and $D u \equiv D v$ in $\Omega$. This completes the proof.

Tam's Theorem is an immediate consequence of Theorem 2 .

Corollary 3. Every solution $u$ of (2) is of the form $\phi_{\beta}+$ constant for some $|\beta|<1$, where $\Omega$ is the infinite strip $\left(-\frac{1}{2}, \frac{1}{2}\right) \times \mathbb{R}$,

$$
\phi_{\beta}=-\frac{1}{\sqrt{1-\beta^{2}}} \sqrt{\left(\frac{1}{2 \cos \alpha}\right)^{2}-x^{2}}+\frac{\beta}{\sqrt{1-\beta^{2}}} y,
$$


$\beta$ satisfies $\int_{\Gamma_{y_{0}}} T \phi_{\beta} \cdot \nu_{1}=\int_{\Gamma_{y_{0}}} T u \cdot \nu_{1}$, for some constant $y_{0}$.

Tam's Theorem for more general domains $\Omega$ is stated in the following Theorem :

TheOREM 4. Let $g_{1}, g_{2} \in C^{1}(\mathbb{R})$ satisfy $g_{2}<g_{1}$ in $\mathbb{R}$. Set $\Omega=\left\{(x, y) \mid g_{2}(y)<x<g_{1}(y)\right\}$. Let $u, v \in C^{2}(\Omega)$ satisfy $T u, T v \in$ $C^{0}(\bar{\Omega}) \cap C^{1}(\Omega)$. Suppose that for every $\varepsilon>0,|D u|$ is uniformly bounded in $\left\{(x, y) \mid g_{2}(y)+\varepsilon \leq x\right.$ and $\left.x \leq g_{1}(y)-\varepsilon\right\}$. And if

$$
\left\{\begin{array}{l}
\operatorname{div} T v=\operatorname{div} T u \\
T v \cdot \nu=T u \cdot \nu \\
\int_{\Gamma_{y_{0}}} T v \cdot \nu_{1} d \sigma=\int_{\Gamma_{y_{0}}} T u \cdot \nu_{1} d \sigma \\
\left|\Gamma_{y}\right|=O\left(|y|^{\frac{1}{3}-\alpha}\right)
\end{array}\right.
$$$$
\text { in } \Omega \text {, }
$$$$
\text { on } \partial \Omega \text {, }
$$$$
\text { for some constant } y_{0} \text {, }
$$$$
\text { as } y \rightarrow \pm \infty
$$$$
\text { for some constant } \alpha
$$$$
\text { with } 0<\alpha<\frac{1}{3} \text {, }
$$

where $\Gamma_{y}=\left[g_{2}(y), g_{1}(y)\right] \times\{y\}$, then we have $u \equiv v+$ constant in $\Omega$.

Proof. The proof is similar to that of Theorem 2. Here we only sketch the proof.

For each $y \in \mathbb{R}$, let us write

$$
f(y)=\int_{\Gamma_{y}} \tan ^{-1}(u-v)(T u-T v) \cdot \nu_{1} .
$$

By (6), $f(y)$ is increasing in $y$, hence $\lim _{y \rightarrow \pm \infty} f(y)$ exist. We claim that $\lim _{y \rightarrow+\infty} f(y)=0$. Otherwise, there exist two positive constants $y_{0}^{\prime}$ and $C_{1}$ such that

$$
|f(y)| \geq C_{1} \text { for every } y \geq y_{0}^{\prime}>0 .
$$

Hence $C_{1} \leq\left|\int_{\Gamma_{y}} \tan ^{-1}(u-v)(T u-T v) \cdot \nu_{1}\right| \leq \pi\left|\Gamma_{y}\right|$, and we have $\left|\Gamma_{y}\right| \geq \frac{C_{1}}{\pi}$ for every $y \geq y_{0}^{\prime}$. Define $m(y)=\frac{1}{\left|\Gamma_{y}^{\prime}\right|} \int_{\Gamma_{y}^{\prime}} \tan ^{-1}(u-v) d \sigma$ for every $y \geq y_{0}^{\prime}$, where $\Gamma_{y}^{\prime}=\left[g_{2}(y)+\frac{C_{1}}{8 \pi}, g_{1}(y)-\frac{C_{1}}{8 \pi}\right] \times\{y\}$.

By hypothesis, $|D u|$ is uniformly bounded in $\bigcup_{y \geq y_{0}^{\prime}} \Gamma_{y}^{\prime}$, so there exists a positive constant $C_{2}$ (independent of $y$ ) such that

$$
|D u| \leq C_{2} \text { in } \bigcup_{y \geq y_{0}^{\prime}} \Gamma_{y}^{\prime}
$$


Hence for each $y \geq y_{0}^{\prime}$, we have

$$
\begin{aligned}
\left|\int_{\Gamma_{y}^{\prime}}\left(\tan ^{-1}(u-v)-m(y)\right)(T u-T v) \cdot \nu_{1}\right| & \\
& \geq|f(y)|-\frac{C_{1}}{2} \geq \frac{|f(y)|}{2} .
\end{aligned}
$$

Applying Poincare inequality to the left hand side of $\left(12^{\prime}\right)$, we obtain

$$
\begin{aligned}
\frac{|f(y)|}{2} & \leq 2 \int_{\Gamma_{y}^{\prime}}\left|\tan ^{-1}(u-v)-m(y)\right| \\
& \leq 2\left|\Gamma_{y}^{\prime}\right| \int_{\Gamma_{y}^{\prime}} \frac{|D u-D v|}{1+(u-v)^{2}} \\
& \leq \frac{C_{1}}{4}+2\left|\Gamma_{y}^{\prime}\right| \int_{\Gamma_{y}^{\prime} \cap\left\{|D u-D v| \geq 8^{-1}\left|\Gamma_{y}^{\prime}\right|^{-2} C_{1}\right\}} \frac{|D u-D v|}{1+(u-v)^{2}} .
\end{aligned}
$$

Hence for every $y \geq y_{0}^{\prime}$, we have

$$
\frac{|f(y)|}{8\left|\Gamma_{y}^{\prime}\right|} \leq \int_{\Gamma_{y}^{\prime} \cap\left\{|D u-D v| \geq 8^{-1}\left|\Gamma_{y}^{\prime}\right|^{-2} C_{1}\right\}} \frac{|D u-D v|}{1+(u-v)^{2}} .
$$

Combining $\left(13^{\prime}\right)$ with the inequalities (3) and (14), we obtain

$$
\begin{gathered}
\frac{|f(y)|}{8\left|\Gamma_{y}^{\prime}\right|} \leq 8\left|\Gamma_{y}^{\prime}\right|^{2} C_{1}^{-1} \sqrt{1+\left(C_{2}+8^{-1}\left|\Gamma_{y}^{\prime}\right|^{-2} C_{1}\right)^{2}}\left(1-\frac{C_{2}}{\sqrt{1+C_{2}^{2}}}\right)^{-1} \\
\cdot \int_{\Gamma_{y}^{\prime} \cap\left\{|D u-D v| \geq 8^{-1}\left|\Gamma_{y}^{\prime}\right|^{-2} C_{1}\right\}} \frac{(D u-D v) \cdot(T u-T v)}{1+(u-v)^{2}} .
\end{gathered}
$$

Since $\Gamma_{y}^{\prime} \subset \Gamma_{y}$,

$$
\begin{aligned}
&\left|\Gamma_{y}^{\prime}\right|^{2} \sqrt{1+\left(C_{2}+8^{-1}\left|\Gamma_{y}^{\prime}\right|^{-2} C_{1}\right)^{2}} \\
& \leq\left|\Gamma_{y}\right|^{2} \sqrt{1+\left(C_{2}+8^{-1}\left|\Gamma_{y}\right|^{-2} C_{1}\right)^{2}},
\end{aligned}
$$

it is easy to see that

$$
\begin{aligned}
|f(y)| \leq & 64\left|\Gamma_{y}\right|^{3} C_{1}^{-1} \sqrt{1+\left(C_{2}+8^{-1}\left|\Gamma_{y}\right|^{-2} C_{1}\right)^{2}} \\
& \cdot\left(1-\frac{C_{2}}{\sqrt{1+C_{2}^{2}}}\right)^{-1} \int_{\Gamma_{y}} \frac{(D u-D v)(T u-T v)}{1+(u-v)^{2}},
\end{aligned}
$$


hence we have

$$
C_{4}\left|\Gamma_{y}\right|^{-3}|f(y)| \leq \int_{\Gamma_{y}} \frac{(D u-D v) \cdot(T u-T v)}{1+(u-v)^{2}}
$$

where

$$
C_{4}=64^{-1} C_{1}\left(1+\left(C_{2}+8^{-1}\left(\frac{C_{1}}{\pi}\right)^{-2} C_{1}\right)^{2}\right)^{-\frac{1}{2}}\left(1-\frac{C_{2}}{\sqrt{1+C_{2}^{2}}}\right)
$$

By Fubini's Theorem, we have

$$
\int_{\Gamma_{y}} \frac{(D u-D v) \cdot(T u-T v)}{1+(u-v)^{2}}=f^{\prime}(y)
$$

Hence

$$
\frac{f^{\prime}(y)}{|f(y)|} \geq C_{4}\left|\Gamma_{y}\right|^{-3} \geq C_{5} y^{-1+3 \alpha} \text { for } y \geq y_{0}^{\prime}>0
$$

where $C_{5}$ is a positive constant. Integrate (17) from $y_{0}^{\prime}$ to $y, y>y_{0}^{\prime}$, we obtain

$$
|\log | f(y)|-\log | f\left(y_{0}^{\prime}\right)|| \geq \frac{C_{5}}{1-3 \alpha}\left(y^{3 \alpha}-y_{0}^{\prime 3 \alpha}\right) .
$$

Note that $|f(y)| \leq \pi\left|\Gamma_{y}\right|=O\left(y^{\frac{1}{3}-\alpha}\right)$. Let $y \rightarrow+\infty$ in (18), we get a contradiction. The remainder of the proof is similar to that of Theorem 2.

REMARK. Let $\Omega$ be a domain (bounded or unbounded) in $\mathbb{R}^{n}$ and let $k$ be a positive constant, where $n \geq 2$ is an integer. Suppose that $\alpha$ is defined on $\partial \Omega$ with $0 \leq \alpha \leq 2 \pi$. Then the boundary value problem

$$
\begin{cases}\operatorname{div} T u=k u & \text { in } \Omega \\ T u \cdot \nu=\cos \alpha & \text { on } \partial \Omega\end{cases}
$$

determines the height $u$ of a capillary surface in a uniform gravitational field. 
It was proved by Finn and the author [5] that if (19) has a solution then it is unique. We point out that neither growth condition of $u$ nor condition on the form of $\Omega$ at infinity is imposed.

\section{REFERENCES}

[1] J.T. Chen and W.-H. Huang, Convexity of capillary surfaces in the outer spaces, Invent. Math., 67 (1982), 253-259.

[2] P. Collin, Deux exemples de graphes courbure moyenne constante sur une bande de $\mathbb{R}^{2}$, C. R. Acad. Sci. Paris, 311 (1990), 539-542.

[3] R. Finn, New estimates for equations of minimal surface type, Arch. Rational Mech. Anal., 14 (1963), 337-375.

[4] - Remarks relevant to minimal surfaces and to surfaces of prescribed mean curvature, J. Analyse Math., 14 (1965), 139-160.

[5] R. Finn and J.-F. Hwang, On the comparison principle for capillary surfaces, J. Fac. Sci. Univ. Tokyo Sect. IA, Math., 36 (1989), 131-134.

[6] J.C.C. Nitsche, Vorlesungen über Minimalfläschen, Springer-Verlag, Berlin-Heidelberg-New York, 1975.

[7] L.F. Tam, On the uniqueness of capillary surfaces without gravity over an infinite strip, Indiana Univ. Math. J., 36 (1987), 79-89.

[8] - On the uniqueness of capillary surfaces, Variational Methods for Free Surfaces Interfaces. (ed. P. Concus and R. Finn) Springer-Verlag, Berlin-Heidelberg-New York, 1987.

[9] A.N. Wang, Constant mean curvature surfaces on a strip, Pacific J. Math., 145 (1990), 395-396.

Received October 29, 1992. Partially supported by grant NSC 82-0208-M-001078 .

INSTITUTE OF MATHEMATICS

ACADEMIa Sinica

TAIPEI 11529

TAIWAN, R.O.C. 



\title{
PACIFIC JOURNAL OF MATHEMATICS
}

Founded by E. F. Beckenbach (1906-1982) and F. Wolf (1904-1989)

\section{EDITORS}

Sun-Yung Alice Chang (Managing Editor)

University of California

Los Angeles, CA 90095-1555

pacific@math.ucla.edu

F. Michael Christ

University of California

Los Angeles, CA 90095-1555

christ@math.ucla.edu

Thomas Enright

University of California

San Diego, La Jolla, CA 92093

tenright@ucsd.edu

Nicholas Ercolani

University of Arizona

Tucson, AZ 85721

ercolani@math.arizona.edu
Robert Finn

Stanford University

Stanford, CA 94305

finn@gauss.stanford.edu

Vaughan F. R. Jones

University of California

Berkeley, CA 94720

vfr@math.berkeley.edu

Steven Kerckhoff

Stanford University

Stanford, CA 94305

spk@gauss.stanford.edu
Martin Scharlemann

University of California

Santa Barbara, CA 93106

mgscharl@math.ucsb.edu

\section{Gang Tian}

Courant Institute

New York University

New York, NY 10012-1100

tiang@taotao.cims.nyu.edu

V. S. Varadarajan

University of California

Los Angeles, CA 90095-1555

vsv@math.ucla.edu

\section{SUPPORTING INSTITUTIONS}

\section{CALIFORNIA INSTITUTE OF TECHNOLOGY \\ NEW MEXICO STATE UNIVERSITY \\ OREGON STATE UNIVERSITY \\ STANFORD UNIVERSITY \\ UNIVERSITY OF ARIZONA \\ UNIVERSITY OF BRITISH COLUMBIA \\ UNIVERSITY OF CALIFORNIA UNIVERSITY OF HAWAII}

\author{
UNIVERSITY OF MONTANA \\ UNIVERSITY OF NEVADA, RENO \\ UNIVERSITY OF OREGON \\ UNIVERSITY OF SOUTHERN CALIFORNIA \\ UNIVERSITY OF UTAH \\ UNIVERSITY OF WASHINGTON \\ WASHINGTON STATE UNIVERSITY
}

The supporting Institutions listed above contribute to the cost of publication of this Journal, but they are not owners or publishers and have no responsibility for its contents or policies.

Manuscripts must be prepared in accordance with the instructions provided on the inside back cover.

The Pacific Journal of Mathematics (ISSN 0030-8730) is published monthly except for July and August. Regular subscription rate: $\$ 215.00$ a year (10 issues). Special rate: $\$ 108.00$ a year to individual members of supporting institutions.

Subscriptions, orders for back issues published within the last three years, and changes of subscribers address should be sent to Pacific Journal of Mathematics, P.O. Box 4163, Berkeley, CA 94704-0163, U.S.A. Prior back issues are obtainable from Kraus Periodicals Co., Route 100, Millwood, NY 10546.

The Pacific Journal of Mathematics at the University of California, c/o Department of Mathematics, 981 Evans Hall, Berkeley, CA 94720 (ISSN 0030-8730) is published monthly except for July and August. Second-class postage paid at Berkeley, CA 94704, and additional mailing offices. POSTMASTER: send address changes to Pacific Journal of Mathematics, P.O. Box 6143, Berkeley, CA 94704-0163.

\author{
PUBLISHED BY PACIFIC JOURNAL OF MATHEMATICS at University of California, \\ Berkeley, CA 94720, A NON-PROFIT CORPORATION \\ This publication was typeset using AMS-LATEX, \\ the American Mathematical Society's TEX macro system. \\ Copyright (C) 1995 by Pacific Journal of Mathematics
}




\section{PACIFIC JOURNAL OF MATHEMATICS}

Volume 169 No. $1 \quad$ May 1995

Minimal sets of periods for torus maps via Nielsen numbers

Lluís AlsedÀ, StewART BALdWin, JAUME Llibre,

RICHARD SWANSON and WIESLAW SZLENK

Diagonalizing Hilbert cusp forms

Timothy ATWILL

A splitting criterion for rank 2 vector bundles on $\mathbf{P}^{n}$

EDOARDO BALLICO

Controlling Tietze-Urysohn extensions

MARC FRANTZ

Length of Julia curves

DAVID H. HAMILTON

On the uniqueness of capillary surfaces over an infinite strip

JENN-FANG HWANG

Volume estimates for log-concave densities with application to iterated 107 convolutions

MARIUS JUNGE

A reflection principle in complex space for a class of hypersurfaces and mappings

FRANCINE ANTOINETTE MEYLAN

Jean Bourgain's analytic partition of unity via holomorphic martingales 161

PAUL F.X. MÜLLER

Characters of Brauer's centralizer algebras 